\title{
CSCI DISTINGUISHED SCIENTIST LECTURE \\ Chromatin remodeling: The interface between extrinsic cues and the genetic code?
}

Shereen Ezzat MD, FRCPC, FACP
Department of Medicine and the Endocrine Oncology Site Group, Princess Margaret Hospital, Ontario Cancer Institute, University Health Network, Toronto, Ontario, Canada M5G-1X5

Presented 25th September, 2008 at CSCI meeting, Toronto Dr. Ezzat was the 2008 CSCI Distinguished Scientist Lecturer

Clin Invest Med 2008; 31 (5): E272-E281.
The successful completion of the human genome project ushered a new era of hope and skepticism. However, the promise of finding the fundamental basis of human traits and diseases appears less than fulfilled. The original premise was that the DNA sequence of every gene would allow precise characterization of critical differences responsible for altered cellular functions. The characterization of intragenic mutations in cancers paved the way for early screening and the design of targeted therapies. However, it has also become evident that unmasking genetic codes alone cannot explain the diversity of disease phenotypes within a population. Further, classic genetics has not been able to explain the differences that have been observed among identical twins or even cloned animals. This new reality has re-ignited interest in the field of epigenetics. While traditionally defined as heritable changes that can alter gene expression without affecting the corresponding DNA sequence, this definition has come into question. The extent to which epigenetic change can also be acquired in response to chemical stimuli represents an exciting dimension in the "nature vs nurture" debate. In this review I will describe a series of studies in my laboratory that illus- trate the significance of epigenetics and its potential clinical implications.

\section{The importance of histone modifications in gene} control

The assembly of DNA into a compact structure termed "chromatin" is essential for packaging the genome into the relatively confining regions of the cell nucleus. The nucleosome, the basic unit of chromatin, consists of $\sim 200$ bp of DNA coiled twice around an octamer, which is composed of dimers of the core histones $\mathrm{H} 1$ to $\mathrm{H} 4$. In a cell, the nucleosome is further compacted into a higher-order structure. Such compaction can result in the regulatory DNA elements of genes becoming inaccessible to regulatory proteins or transcription factors, thereby limiting gene expression and resulting in gene silencing (Fig 1). Histones can be modified by covalent modifications through phosphorylation or ribosylation. However, acetylation and methylation represent the most widely studied dynamic processes. In particular, Acetylation (Ac) of specific lysine $(\mathrm{K})$ residues on histone $\mathrm{H} 4$ and methylation (Me) of lysine 9 (K9) on $\mathrm{H} 3$ can be found 


\section{Silenced gene}

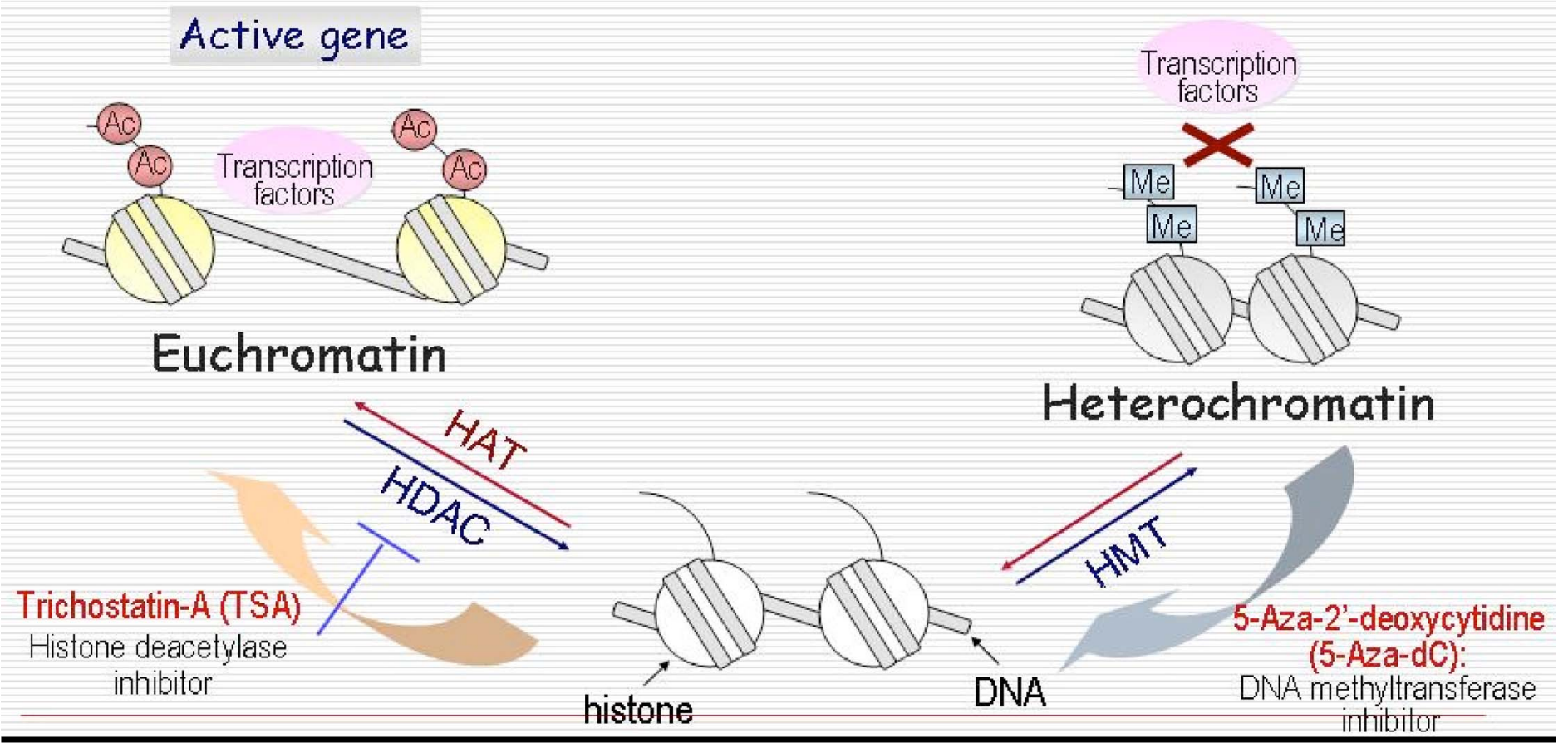

FIGURE 1. Enzymatic modifications of histone tails influences gene expression by altering DNA packaging. DNA in the nucleus is wrapped tightly around histone cores (circles) which can exist in an open conformation (relaxed) euchromatin phase (left). In this state, DNA binding sites are readily accessible for nuclear proteins to gain access and function as transcription factors. In the more restricted (right; heterochromatin) closed state, histone tails are modificatied through methylation a process which typically creates a more restricted environment thus preventing access of transcription factors. These enzymatic reactions are catalyzed by histone acetylation transferase (HAT) or histone deacetylation (HDAC) that are regarded as dynamic, reversible reactions subject to chemical and environmental stimuli.

at inactive gene loci. In contrast, acetylation on K9 and $\mathrm{K} 14$ of $\mathrm{H} 3$, methylation on $\mathrm{K} 4$ of $\mathrm{H} 3$, or acetylation on $\mathrm{K} 5$ of $\mathrm{H} 4$ can be identified at gene loci that are either active or have the potential to become activated (Fig. 1). Increasingly, the enzymes involved in specific dynamic histone modifications are being discovered. What follows is our identification of the role of such a protein in mouse and human physiology.

\section{The importance of Ikaros in hematopoietic stem cells}

Gene targeting experiments have firmly established that nuclear factors encoded by the Ikaros gene are essential for normal lymphoid development. ${ }^{1}$ Mice homozygous for a null mutation in Ikaros display deficient B- and T-cell differentiation ${ }^{2,3}$, whereas those homozygous for a dn mutant form of Ikaros lack all lymphocytes. ${ }^{4}$ The more severe phenotype of the $\mathrm{dn}$ Ik-mutant suggested the presence of Ikaros homologues, leading to the identification of the related factors Aiolos ${ }^{5}$, Helios ${ }^{6,7}$, and Eos. ${ }^{1}$ In addition to its role in differentiation, Ikaros plays an important role in regulating cell proliferation. Ik+/- mice exhibit hyperactive $\mathrm{T}$ cell receptor-mediated proliferative responses and eventually develop leukemias and lymphomas. ${ }^{8,9}$ Animals heterozygous for the dn Ik- 6 that lacks the DNA-binding domain develop T-cell lymphoproliferative disorders similar to human $\mathrm{T}$ lymphoblastic leu- 
kemia or lymphoma, presumably as a result of inactivation of the normal allele. ${ }^{8}$ Thus, Ikaros appears to be an essential regulator of chromatin structure affecting early lymphocyte differentiation as well as differentiated cell expansion.

\section{The role of Ikaros in the anterior pituitary gland}

My clinical and research interests as an endocrinologist have focused on the pituitary gland. This is based on the fact that this gland regulates many of the functions of other endocrine glands and their target tissues throughout the body. There is virtually no organ or tissue that is not affected, directly or indirectly, by the hormones that are secreted by the anterior and posterior pituitary. Pituitary dysfunction is common, however, the manifestations of pituitary disease vary from florid clinical features that can readily be identified and recognized as pituitary in etiology, to more subtle and nonspecific problems that are often overlooked or misdiagnosed, leaving the relationship to pituitary malfunction unnoticed. The former, diseases such as acromegaly and Cushing's disease, are rare, hence the misconception that pituitary disease is rare.

The process of anterior pituitary cell development and differentiation follows a highly specific pattern and temporal sequence. Several putative transcriptionregulating proteins have been identified in the pituitary and have been implicated as key elements in the definition of cell specific phenotypes and the regulation of hormone gene expression. ${ }^{10-12}$ Which of the lineage-regulating factors predominates in determining the lineage choice and expansion can be decided not only by the expression and relative concentration of the transcription factor but also by the chromatin accessibility at their cognate sites.

As mentioned above, Ikaros was originally described as a transcription factor that binds to regulatory sequences of genes expressed in lymphoid cells. ${ }^{13,14}$ The single copy gene contains seven exons that can, by alternative splicing, give rise to eight isoforms. The $\mathrm{N}$-terminal region includes a domain with zinc finger motifs critical for DNA-binding. Dominant-negative $(\mathrm{dn})$ forms of Ikaros lack the DNA-binding domain. The various isoforms can act either as transcriptional activators or repressors as part of an integral component of a functionally diverse chromatin remodeling network. ${ }^{13}$ It has become readily evident that the Ikaros system represents an example of a finely tuned system with distinct regulatory functions, particularly during early developmental stages in restricted target tissues.

In the next section of this review I will cover the evidence implicating Ikaros as a key factor whose transcriptional actions and chromatin remodeling properties direct hypothalamic neuroendocrine and pituitary cell population expansion during development. We propose that the governing mechanisms involved in the regulation and action of Ikaros are of importance during developmental as well as neoplastic transitions.

\section{Ikaros directs anterior pituitary cell expansion}

My laboratory's studies focused on characterization of the striking phenotype of the Ikaros-deficient mouse model. In particular, we found that deletion of both copies of this gene result in profound dwarfism with nearly $95 \%$ mortality by 6 -weeks of age. ${ }^{15}$ Animals losing one copy of this gene (Ik+/Ik-) survive for 4-5 months and subsequently develop lymphoproliferative disorders with leukemic or atypical lymphoid infiltrates of several tissues. Although these animals were thought to develop infection with sepsis, complete autopsy examinations of our mice have not identified evidence of infection or sepsis. Rather, they show wasting with features of a profound metabolic/ endocrine syndrome. Tissue and blood cultures were found to be negative. These animals have normal myeloid marrow elements, and there is no evidence of a neutrophilic response to infection. Moreover, bone marrow transplantation or reconstitution of Ikarosdeficient animals after birth with wild-type marrow demonstrated no measurable impact on size and over- 
all health attributable to successful lymphocyte repopulation, supporting the hypothesis of an endocrine/ metabolic basis for the phenotype. ${ }^{15}$ These experiments provided compelling evidence for a lymphocyte-independent role for Ikaros in governing neuroendocrine system development.

\section{Ikaros modulates pituitary cell survival}

The Ikaros gene undergoes alternative splicing, leading to eight different isoforms. ${ }^{16-18}$ Ikaros isoforms differ in the number of N-terminal DNA-binding zinc fingers that differentiates them into members with or without DNA binding properties. ${ }^{16-18}$ All Ikaros isoforms share a common C-terminal domain that contains a transcription activation motif and two zinc finger motifs required for hetero- and homodimerization among the Ik isoforms and for interactions with other proteins. ${ }^{7,8,17} \mathrm{Ik} 1-3$ contain the 3 or more amino $(\mathrm{N})$ terminal zinc fingers that confer high-affinity binding to an Ikaros-specific core DNA sequence motif in the promoters of target genes. ${ }^{17}$

Animals heterozygous for dominant-negative (dn) forms of Ikaros (isoforms which lack the DNA binding domain) develop T-cell disorders similar to human T lymphoblastic leukemia or lymphoma, presumably by inactivation of the normal Ikaros allele. ${ }^{8}$ Overexpression of a dn non-DNA binding isoform Ik6 has been identified in a third of cases of B-cell acute leukemias. ${ }^{19}$ Ikaros mutant mice display a decrease in expression of flk-2 and c-kit receptors which may in part contribute to the early lymphopoietic phenotypes manifested in the absence of Ikaros. ${ }^{20}$

Having identified that Ikaros is expressed in the normal pituitary, we then examined its expression in neoplastic tissues. We detected Ik 1 and $I k 2 / 3$ protein isoforms in human and mouse pituitaries. ${ }^{21} \mathrm{We}$ also identified Ik6 expression in nearly $50 \%$ of human pituitary adenomas. ${ }^{21}$ Forced introduction of the dominant negative form of Ikaros resulted in histone 3 acetylation with activation of the survival Bcl-XL signal. $^{22}$

\section{Ikaros promotes anterior pituitary cell differentiation} through cellular lipids

To further determine the mechanisms of action of Ikaros in pituitary cell growth and differentiation, we used a cDNA microarray strategy. This approach interrogates several thousand genes for changes in expression in response to experimental manipulation. In this instance, by forcing the expression of Ikaros in pituitary cells we uncovered that mediators of cholesterol uptake including the low-density lipoprotein receptor (LDL-R) and sterol-regulatory element binding protein 2 (SREBP2) could be putative targets of $\mathrm{Ik}$ action. ${ }^{23}$ Indeed, we found that Ikaros regulates the low-density lipoprotein receptor (LDL-R) to alter metabolism in pituitary corticotroph cells. The DNAbinding Ikaros isoform, Ik1, binds and enhances activity of the LDL-R promoter. Ik 1 decreases methylation and increases acetylation of histone $\mathrm{H} 3$ lysine 9 at the LDL-R promoter. ${ }^{23}$ Confocal microscopy and quantitative fluorometry demonstrated enhanced LDLcholesterol endocytosis in Ik1-cells that exhibited abundant endoplasmic reticulum, large Golgi complexes, and prominent secretory granule formation, consistent with more robust cholesterol incorporation into functionally relevant membrane-rich organelles. Consistent with these data, LDL-R ${ }^{-/}$mice, like $\mathrm{Ik}^{-/-}$ mice, have decreased circulating levels of adrenocorticotropic hormone. ${ }^{23}$ These findings expand the repertoire of Ikaros actions to include regulation of the cholesterol uptake metabolic pathway. This novel link between tumor suppression and differentiation provides a relationship between cellular metabolism and cancer, and has therapeutic implications for lipidmodifying drugs in Ikaros-associated disorders.

\section{Ikaros directs corticomelanotroph population expansion}

To quantify the in vivo effects of Ikaros on hypothalamic-pituitary development, we examined in detail the effects of disruption of Ikaros on hypothalamic-pituitary architecture, cell number and 
area by quantitative morphometric analyses, proliferation rates and apoptotic indices. Morphologic and morphometric examination of these animals reveals pituitary hypoplasia and dysgenesis in Ik-null mice. During fetal development there is delayed progression of pituitary development with small but architecturally normal pituitaries compared with wild-type littermates that have normal pituitary development. ${ }^{24}$ After birth, the Ik-null mice continue to exhibit a marked reduction in pituitary mass. They have normal cytodifferentiation with the presence of GH-positive somatotrophs, PRL-positive lactotrophs, ACTH-positive corticotrophs, TSH-reactive thyrotrophs and gonadotrophs containing FSH and LH. However, there are marked reductions in the number of adenohypophysial cells, most strikingly involving the ACTH and GHproducing populations. The pituitaries of homozygote mice exhibited a striking reduction of melanocorticotropes that was most marked in the intermediate lobe. The heterozygote and homozygote animals showed no evidence of the classical expansion of the melanocorticotropes that normally proliferate to occupy the entire intermediate lobe.

To specifically examine the functional consequences of disrupted Ikaros signaling on pituitary corticomelanotroph development, we compared the hormonal and developmental profiles of Ik-null mice with their heterozygote and wild-type littermates. ${ }^{15}$ Homozygote Ik-null mice demonstrated the lowest levels of ACTH in the systemic circulation. Levels of the POMC-derived melanocyte-stimulating hormone (MSH) were also reduced in heterozygote but more strikingly in Ik-null mice. The functional consequences of a diminished population of POMCproducing corticomelanotroph cells were most evident in homozygote mice. Consistent with the trophic functions of $\mathrm{ACTH}$ on adrenocortical development and function, Ik-null mice display reduced circulating corticosterone levels compared with heterozygote and wild-type littermates. These changes were also reflected by reduction of adrenal gland size with striking loss of adrenocortical mass, especially in homozygous Ik-deficient mice.

To examine the possibility that the diminished POMC production and adrenocortical insufficiency in Ikaros-deficient animals may be mediated through altered cytokine production by a dysregulated lymphocyte population, we examined the impact of wild-type bone marrow reconstitution on the endocrine phenotype. Five weeks after adoptive bone marrow transfer in neonatal mice, the proportion of splenic $\mathrm{CD}^{+}$and $\mathrm{CD}^{+} \mathrm{T}$-cells in the homozygous Ik-null recipients of Ik wild-type syngenic bone marrow was significantly restored compared to vehicle-treated controls. Despite successful reconstitution with normal lymphocytes, the phenotype of homozygote Ik-null animals was unchanged with persistently small pituitaries and reduction in POMC expression that was indistinguishable from vehicle-treated age-matched homozygote animals. These data provide evidence for a direct role of Ikaros in pituitary corticomelanotroph development and function independent of its influence on lymphopoiesis.

Having demonstrated that Ikaros mutant mice suffer from adrenocortical insufficiency, we examined the impact of systemic hormone replacement on growth and survival of the Ik-null mouse. Glucocorticoid hormone treatment compared with vehicle alone resulted in significant weight increase in Ik-null mice; the effect was also present, but less evident, in heterozygote animals. By comparison, the same treatment displayed no significant impact on the growth of wildtype animals.

Overall, homozygote Ik-null mice display diminished life expectancy with nearly $5 \%$ mortality at birth and $95 \%$ by 6 weeks of age. Given the recognized functions of Ikaros on immune cell development and function, we performed complete organ surveys in search of opportunistic infections or malignancy. However, we found no evidence of either of these conditions to explain the observed mortality at these stages of development. Moreover, hormone treatment with glucocorticoids resulted in improved survival. In 
particular, Ik-null mice treated with the adrenocortical hormone demonstrated $100 \%$ survival beyond 6 weeks of age. For comparison, none of the vehicletreated homozygote mice survived beyond the same time point.

\section{Ikaros binds and activates the pro-opiomelanocortin (POMC) gene}

To determine the mechanism of Ikaros action responsible for the corticotroph-insufficient phenotype, we analyzed the rat POMC promoter at five potential Ikbinding sites within the -543 bp promoter. ${ }^{15}$ The importance of these putative Ikaros-binding sites was examined by electromobility shift assay (EMSA) and luciferase reporter assays. Two of the three sites which formed specific super-shifted complexes [fragment I (-451/-420) and fragment II (-163/-137)] were identified to be functionally significant as determined through mutational analyses and co-transfection studies. ${ }^{15}$ These Ikaros-binding sites in the POMC promoter are within close proximity ( $\sim 70 \mathrm{bp})$ upstream and downstream of the recently identified Tpit/ Pitx1 regulatory element. ${ }^{25}$ The T-box factor Tpit is restricted to POMC-expressing corticomelanotroph pituitary cells. ${ }^{25}$ It activates POMC transcription in cooperation with contiguously bound Pitx 1 by recruiting the SRC/p160 co-activators. ${ }^{26}$ Given the critical role of Ikaros in nuclear dimerization, the potential for physical interaction between Ikaros and Tpit needs to be specifically examined. Nevertheless, forced Ikaros expression, but not ( $\mathrm{dn}$ ) Ik6, results in induction of the endogenous POMC gene at the mRNA and protein levels. ${ }^{15}$

\section{The role of Ikaros in the hypothalamus}

\section{Ikaros directs somatotroph expansion}

Ikaros-deficient animals display a dwarf phenotype with body weight reaching only $\sim 50 \%$ of their wildtype littermates. This dwarfism is associated with diminished growth hormone $(\mathrm{GH})$ secretion, as evi- denced by a reduction of the GH-target growth factor IGF-I by $>50 \%$ reduction in homozygote animals. The Ikaros heterozygous animals display near normal somatic growth with more modest $(\sim 12 \%)$ reductions in IGF-I levels compared to wild-type mice. Healthy homozygote mice had the same body proportions as those of heterozygote and wild-type mice. At autopsy, internal organs were proportionally equivalent across the genotypes with the exception of the contracted pituitary and adrenal glands of homozygote mice. These features of GH deficiency are not a result of nonspecific generalized pituitary mal-development, since the animals have normal or slightly elevated circulating levels of TSH and prolactin. Although these animals exhibit glucocorticoid deficiency that could account for $\mathrm{GH}$ insufficiency, glucocorticoid replacement that significantly improved viability resulted in only a minimal increase in body weight. ${ }^{15}$

The changes of proportionate postnatal dwarfism are indicative of GH deficiency in homozygous Iknull mice. Again, to exclude the possibility of a secondary response to immunodeficiency, we performed bone marrow reconstitution studies and showed that hematopoietic replacement does not alter IGF-I levels and restore normal growth. We carried out administration of exogenous $\mathrm{GH}$ with a response of circulating IGF-I levels and body weight reflecting somatic growth. ${ }^{27}$

The pituitary somatotrope population displayed quantitative differences across the Ikaros genotypes during early postnatal development. Somatotrophs were present in approximately the same proportion of cells in all genotypes, but the glands were smaller in heterozygotes than in wild-type mice and even smaller in homozygotes. The intensity of GH reactivity was also reduced in homozygote mice. GH positivity persisted in the intermediate lobe of the lateral wings, likely attributable to the lack of melanocorticotrope expansion that normally replaces intermediate lobe somatotropes during development. Other cell types were not measurably affected; the number and area of immunoreactive thyrotrophs and gonadotrophs were 
not disproportionately different from those of wildtype littermates. ${ }^{27}$

\section{Ikaros reciprocally regulates growth hormone and prolactin gene expression}

To examine the regulation of GH by Ikaros, we examined pituitary mammosomatotroph GH4 cells that express abundant Ikaros. We found that Ikaros reciprocally regulates the GH and PRL genes. ${ }^{28}$ In contrast to the expected results, we documented that wild-type Ikaros (Ik1) inhibits GH mRNA and protein expression while stimulating PRL mRNA and protein levels. Ikaros does not bind directly to the proximal (-360) GH promoter. Instead, Ikaros significantly abrogates the effect of the histone deacetylation inhibitor trichostatin-A on this promoter. Ikaros selectively deacetylates histone 3 residues on the proximal transfected or endogenous GH promoter and limits access of the Pit 1 activator. In contrast, Ikaros acetylates histone 3 on the proximal PRL promoter and facilitates Pit1 binding to this region in the same cells (28). These data provide evidence for Ikaros mediatedhistone acetylation and chromatin remodeling in the selective regulation of pituitary GH and PRL hormone gene expression in mammosomatotroph cells.

\section{Ikaros regulates hypothalamic GHRH development}

This paradoxical result did not explain the dwarf phenotype of the Ik-null mice and therefore another explanation for GH-deficiency was sought in the hypothalamus. Abundant levels of Ikaros expression were identified in the hypothalamus of wild-type littermates. This staining was localized predominantly within the ventral hypothalamus and was colocalized with GHRH in hypothalamic neurons. ${ }^{27}$ The number of positive cells reaches a maximum at embryonic day (e)18 and shows a reduction in newborn mice; only scattered cells are positive in the adult brain. Ik-null mice showed a striking lack of hypothalamic GHRH. Over-expression of Ikaros enhanced GHRH promoter activity and induced endogenous GHRH gene expression, proving that there is a critical role of Ikaros in the functional regulation of GHRH. ${ }^{27}$ These data unmask a wider role for Ikaros in the neuroendocrine system, highlighting a critical contribution to the development of the hypothalamic-pituitary somatotrophic axis.

\section{The role of Ikaros in higher central nervous system functions}

We recently also found that Ikaros transcription factors are selectively present in the brain within the developing striatum. In one other study focusing on the relevance of $\mathrm{Ik}$ to the enkephalinergic system ${ }^{29}$ the authors concluded that Ik is co-expressed with enkephalin mRNA and may act as a positive regulator of enkephalinergic specification in the developing striatum. While enkephalin is one of several striatal cotransmitters, expressed by a subset of medium spiny neurons (MSN), there are many others, including GABA, substance $\mathrm{P}$ and dopamine D1 and D2 receptors. ${ }^{30}$ We found Ik in precursor cells of several neurostriatal compartments suggesting that it may play a role as a general maturation factor for medium spiny neurons.

With these neuro-localization studies in mind, we proceeded to systematically examine the impact of loss of Ikaros on neurostriatal-mediated functions. We performed a battery of standardized neurobehavioral tests including the elevated plus-maze (a measure of anxiety-like behaviour), the acoustic startle response and pre-pulse inhibition tests (measures of motor and autonomic reaction), the pinch test (a measure of catalepsy), and contextual fear conditioning (measures of learning and emotion). None of these behavioural functions were significantly altered in Ik-null mice, however, it was specifically in the Porsolt's forced swim test (a measure of depression-like behavior) where we found $\mathrm{Ik}^{-/-}$mice spending significantly less time in immobility than their $\mathrm{Ik}^{+/+}$littermates ${ }^{31}$. This phenotype is consistent with reduced behavioral despair. These findings suggest that Ikaros-mediated 


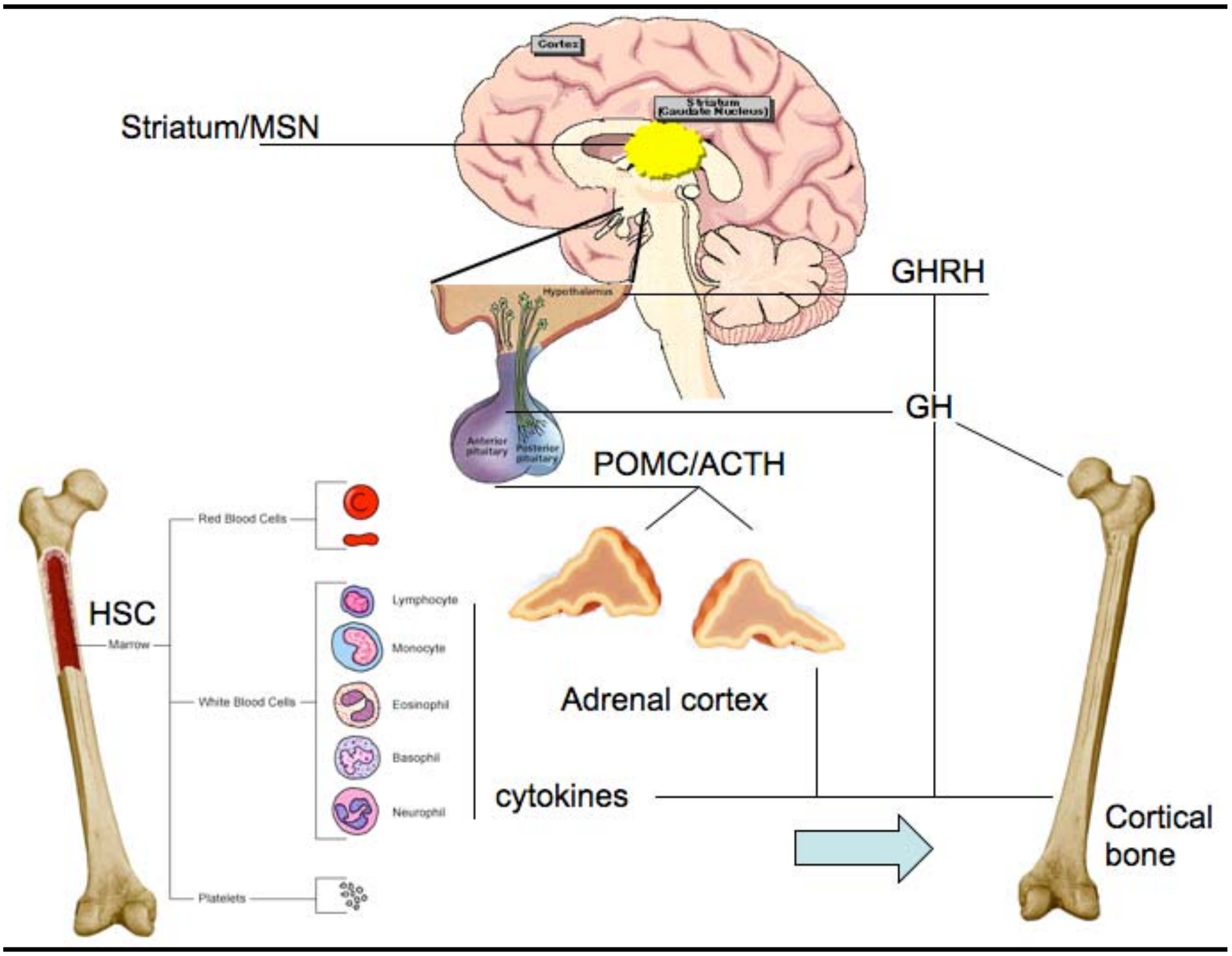

FIGURE 2. Ikaros is present in restricted regions of the neuroendocrine and hematopoietic systems. In the brain: the highest expression is in the medium spiny neurons (MSN) of the striatum where loss of function results in neurobehavioural changes characterized by an anti-depressant phenotype. In the hypothalamus: the median eminence of GHRH-containing neurons co-localize with Ikaros expression. Loss of Ikaros severely diminishes GHRH production and consequently growth hormone and its target insulin-like growth factor-I (IGF-I). In the anterior pituitary gland: Ikaros is expressed in corticotrophs which govern the adrenocorticotrophic axis. Ikaros is also expressed in the somatotrophs where it plays a direct inhibitory role. In the hematopoietic system: Ikaros' peak expression is in stem cells where it directs lineage commitment along the lymphoid pathway. The multidimensional actions of Ikaros serve to sort and integrate diverse signals to govern neuro-endocrine-immune interactions.

neuro-striatal cytodifferentiative functions impose significant and selective impact on depressive behaviour. ${ }^{31}$ The neurochemical Ikaros targets involved in mediating these functions will undoubtedly provide novel pharmacologic opportunities for the treatment of psycho-affective disorders.

\section{Concluding remarks}

Our identification of Ikaros as a transcriptional regulator of the pituitary hormones and consequently the adrenal axis, its epigenetic control of the growth hormone axis, and the exciting new finding of Ikaros in specific hypothalamic and corticostriatal neurons that 
determine affective behaviour, raises new questions about the pleiotropic neuroendocrine functions of this zinc finger protein (Figure 2). The established functions of Ikaros as a mediator of early and late hematopoietic stem cell commitment provide further insights into the role of Ikaros as an integrator of endocrine, immune and neurobehavioural functions. The studies reviewed here indicate that Ikaros can influence hypothalamic/pituitary cell development, differentiation, and proliferation, and provide evidence that these effects are mediated through overlapping and redundant cellular and nuclear targets. The balance between Ikaros and its dominant negative isoforms appear to regulate multiple control elements of genes through covalent histone acetylation and methylationdependent mechanisms. The net influence of these interactions serves to shape a chromatin environment that permits recruitment of an activating or repressing complex essential for gene-specific control. We hope that these findings bring us closer to the interface between environment influences and the genetic code.

\section{Acknowledgements}

The author wishes to thank members of the Ezzat lab for their hard work and contribution to the studies reviewed, with special thanks for the unwavering support of his long-time collaborator and partner, Dr. Sylvia Asa. Work reviewed here was supported by operating research funding from the Canadian Institutes of Health Research.

\section{References}

1. Cortes M, Wong E, Koipally J, Georgopoulos K. Control of lymphocyte development by the Ikaros gene family. Curr Opin Immunol 1999;11:167-71.

2. Wang JH, Nichogiannopoulou A, Wu L et al. Selective defects in the development of the fetal and adult lymphoid system in mice with an Ikaros null mutation. Immunity 1996;5:537-49.

3. Winandy S, Wu L, Wang JH, Georgopoulos K. Pre-T cell receptor (TCR) and TCR-controlled checkpoints in $\mathrm{T}$ cell differentiation are set by Ikaros. J Exp Med 1999;190:1039-48.
4. Georgopoulos K, Bigby M, Wang JH et al. The Ikaros gene is required for the development of all lymphoid lineages. Cell 1994;79:143-56.

5. Morgan B, Sun L, Avitahl N et al. Aiolos, a lymphoid restricted transcription factor that interacts with Ikaros to regulate lymphocyte differentiation. EMBO J 1997;16:2004-13.

6. Kelley CM, Ikeda T, Koipally J et al. Helios, a novel dimerization partner of Ikaros expressed in the earliest hematopoietic progenitors. Curr Biol 1998;8:508-515.

7. Hahm K, Cobb BS, McCarty AS et al. Helios, a T cellrestricted Ikaros family member that quantitatively associates with Ikaros at centromeric heterochromatin. Genes Dev 1998;12:782-96.

8. Winandy S, Wu P, Georgopoulos K. A dominant mutation in the Ikaros gene leads to rapid development of leukemia and lymphoma. Cell 1995;83:289-99.

9. Avitahl N, Winandy S, Friedrich C, Jones B, Ge Y, Georgopoulos K. Ikaros sets thresholds for T cell activation and regulates chromosome propagation. Immunity 1999;10:333-43.

10. Asa SL, Ezzat S. The cytogenesis and pathogenesis of pituitary adenomas. Endocr Rev 1998;19:798-827.

11. Asa SL, Ezzat S. Molecular determinants of pituitary cytodifferentiation. Pituitary 1999;1:159-68.

12. Asa SL, Ezzat S. The pathogenesis of pituitary tumours. Nat Rev Cancer 2002;2:836-49.

13. Molnar A, Wu P, Largespada DA et al. The Ikaros gene encodes a family of lymphocyte-restricted zinc finger DNA binding proteins, highly conserved in human and mouse. J Immunol 1996;156:585-92.

14. Georgopoulos K, Winandy S, Avitahl N. The role of the Ikaros gene in lymphocyte development and homeostasis. Annu Rev Immunol 1997; 15:155-76.

15. Ezzat S, Mader R, Yu S, Ning T, Poussier P, Asa SL. Ikaros integrates endocrine and immune system development. J Clin Invest 2005;115:1021-9.

16. Molnar A, Georgopoulos K. The Ikaros gene encodes a family of functionally diverse zinc finger DNAbinding proteins. Mol Cell Biol 1994;14:8292-303.

17. Sun L, Liu A, Georgopoulos K. Zinc finger-mediated protein interactions modulate Ikaros activity, a molecular control of lymphocyte development. EMBO J 1996;15:5358-69.

18. Hahm K, Ernst P, Lo K, Kim GS, Turck C, Smale ST. The lymphoid transcription factor LyF-1 is encoded by specific, alternatively spliced mRNAs derived from the Ikaros gene. Mol Cell Biol 1994;14:7111-23.

19. Nakase K, Ishimaru F, Avitahl N et al. Dominant negative isoform of the Ikaros gene in patients with adult Bcell acute lymphoblastic leukemia. Cancer Res 2000:60:4062-5. 
20. Nichogiannopoulou A, Trevisan M, Neben S, Friedrich C, Georgopoulos K. Defects in hemopoietic stem cell activity in Ikaros mutant mice. J Exp Med 1999;190:1201-14.

21. Ezzat S, Yu S, Asa SL. Ikaros isoforms in human pituitary tumors: distinct localization, histone acetylation, and activation of the 5 ' fibroblast growth factor receptor-4 promoter. Am J Pathol 2003;163:1177-84.

22. Ezzat S, Zhu X, Loeper S, Fischer S, Asa SL. TumorDerived Ikaros 6 Acetylates the Bcl-XL Promoter to Up-Regulate a Survival Signal in Pituitary Cells. Mol Endocrinol 2006;20:2976-86.

23. Loeper S, Asa SL, Ezzat S. Ikaros Modulates Cholesterol Uptake: A Link Between Tumor Suppression and Differentiation. Cancer Res 2008;68:3715-23.

24. Shewchuk BM, Asa SL, Cooke NE, Liebhaber SA. Pit1 binding sites at the somatotrope-specific DNase I hypersensitive sites I, II of the human growth hormone locus control region are essential for in vivo hGH-N gene activation. J Biol Chem 1999;274:35725-33.

25. Lamolet B, Pulichino AM, Lamonerie T et al. A pituitary cell-restricted $\mathrm{T}$ box factor, Tpit, activates POMC transcription in cooperation with Pitx homeoproteins. Cell 2001;104:849-59.

26. Maira M, Couture C, Le Martelot G, Pulichino AM, Bilodeau S, Drouin J. The T-box factor Tpit recruits SRC/p160 co-activators and mediates hormone action. J Biol Chem 2003;278:46523-32.

27. Ezzat S, Mader R, Fischer S, Yu S, Ackerley C, Asa SL. An essential role for the hematopoietic transcrip- tion factor Ikaros in hypothalamic-pituitary-mediated somatic growth. Proc Natl Acad Sci U S A 2006;103:2214-9.

28. Ezzat S, Yu S, Asa SL. The zinc finger Ikaros transcription factor regulates pituitary growth hormone and prolactin gene expression through distinct effects on chromatin accessibility. Mol Endocrinol 2005;19:1004-11.

29. Agoston DV, Szemes M, Dobi A et al. Ikaros is expressed in developing striatal neurons and involved in enkephalinergic differentiation. J Neurochem 2007; 102:1805-16.

30. Holt DJ, Graybiel AM, Saper CB. Neurochemical architecture of the human striatum. J Comp Neurol 1997;384:1-25.

31. Kiehl TR, Fischer SE, Ezzat S, Asa SL. Mice lacking the transcription factor Ikaros display behavioral alterations of an anti-depressive phenotype. Exp Neurol 2008;211:107-14.

Correspondence to:

Dr. Shereen Ezzat

Ontario Cancer Institute

610 University Ave. \#8-327

Toronto, Ontario, Canada, M5G-2M9

E.mail: shereen.ezzat@utoronto.ca 